\title{
Fine structure of the asymptotic expansion of cyclic integrals
}

\author{
K. K. Kozlowski 1
}

\begin{abstract}
The asymptotic expansion of $n$-dimensional cyclic integrals was expressed as a series of functionals acting on the symmetric function involved in the cyclic integral. In this article, we give an explicit formula for the action of these functionals on a specific class of symmetric functions. These results are necessary for the computation of the $\mathrm{O}(1)$ part in the long-distance asymptotic behavior of correlation functions in integrable models.
\end{abstract}

\footnotetext{
${ }^{1}$ Laboratoire de Physique, ENS Lyon et CNRS, France, karol.kozlowski@ens-lyon.fr
} 


\section{Introduction}

There is a great interest in obtaining exact representations for the correlation functions of integrable models in one-dimensional quantum mechanics[7, 2, 11, 12]. This is especially due to the recent development of experimental techniques allowing one to directly observe such models. Among experimentally interesting correlation functions, one could mention the dynamical ones that are for instance measured through neutron scattering on anti-ferromagnets. It was the determinant representation for the form factors of the spin- $1 / 2 \mathrm{XXZ}$ chain [12] or the non-linear Schrodinger model [13] that opened the possibility for an effective numerical study of these dynamical correlation functions in relatively long chains [5] and diffuse lattice Bose gases [4] (this should be put in contrast with numerical diagonalization that is limited to twenty or so sites). One had to resort to numerics so as to obtain a satisfactory answer as, in general, the exact representations for the correlation functions in integrable models are involved and often not computable in a closed form. Even if simple closed expressions for the correlators do not exist, it is sometimes possible to built on these explicit answers so as to derive the asymptotic behavior of certain correlation functions. This, in turn, allows one to test the conformal field theoretic predictions [3] for the power-law decay with the distance of two-point functions in gapless one-dimensional quantum Hamiltonians [1, 6]. This last program has been carried recently by the author and his collaborators [9]. There, it was shown that it is possible to extract, starting from first-principle based computations on the XXZ spin- $1 / 2$ chain, the long-distance $x$ power-law decay of the longitudinal spin-spin $\left\langle\sigma_{1}^{z} \sigma_{x}^{z}\right\rangle$ correlation function. As expected, these asymptotics were found to match the conformal field theoretic predictions for this quantity.

We now briefly outline the method used for deriving these asymptotics. This will settle the context of the results presented in this paper. The method for extracting the asymptotic behavior of spin-spin correlation functions builds on the master equation [8, 11]. It is an $N$-fold contour integral representation for the generating function $\left\langle\mathrm{e}^{\beta Q_{x}}\right\rangle$ of two-point functions. Starting from this master equation, one should first reach the conformal regime of the model. The latter is supposed to manifest itself in the case of a gapless spectrum above the ground state. For integrable models, this corresponds to sending the size of the model to infinity and restricting oneself to a given range of the coupling constants. In particular, to reach this limit, one should send the number of integrals $N$ to infinity. However, the latter limit is impossible directly in the aforementioned integral representation. To do so, one has first to expand the $N$-fold contour integral into a series, whose summand $a_{n}$ of order $n$ is a linear combination of $2 n$-fold integrals. Each of these integrals depends on the large parameter $x$ and is expressed as products of the so-called cyclic integrals:

$$
\mathcal{I}_{n}\left[\mathcal{F}_{n}\right]=\int_{\Gamma([-q ; q])} \frac{\mathrm{d}^{n} z}{(2 i \pi)^{n}} \int_{-q}^{q} \frac{\mathrm{d}^{n} \lambda}{(2 i \pi)^{n}} \mathcal{F}_{n}\left(\begin{array}{c}
\{\lambda\} \\
\{z\}
\end{array}\right) \prod_{k=1}^{n} \frac{\mathrm{e}^{i x\left[p\left(z_{k}\right)-p\left(\lambda_{k}\right)\right]}}{\left(\lambda_{k}-z_{k}\right)\left(\lambda_{k}-z_{k+1}\right)}
$$

The functions $\mathcal{F}_{n}$ and $p$ are specific of the considered model. The asymptotic behavior of (1.1) drives the one of $a_{n}$. It was shown in [10] that the asymptotic expansion (AE) of cyclic integrals can be deduced from the asymptotic solution of a Riemann-Hilbert problem related to the generalized sine kernel. This AE is written as a series of functionals $I_{n}^{(N, m)}$ acting on the function $\mathcal{F}_{n}$. In order to deduce the AE of $\left\langle\mathrm{e}^{\beta Q_{x}}\right\rangle$, one should replace every cyclic integral in $a_{n}$ by its $\mathrm{AE}$ and then re-order an re-sum the resulting terms. Due to the product structure of $a_{n}$ such a procedure involves summing-up series of products of functionals $I_{n}^{(N, m)}$. In the intermediate steps of this summation procedure, it happens that the $x$-independent function one started with, acquires an $x$-dependence. It thus follows that one has to know how to evaluate the action of the functionals $I_{n}^{(N, m)}$ on certain classes of $x$ dependent functions. This last topic constitutes the subject of this article. It seems rather difficult to give an explicit formula for $I_{n}^{(N, m)}\left[\mathcal{F}_{n}\right]$ when $\mathcal{F}_{n}$ is a generic symmetric function. Indeed, the functionals $I_{n}^{(N, m)}$ have been built rather implicitly through a recursive procedure. However, we found that it is possible to write a simple explicit formula for the leading order in $x$ part of the action of $I_{n}^{(N, m)}$ when focusing on a specific class of $x$-dependent 
functions. Such a result should not be understood as some part of the asymptotics of $2 n$-fold cyclic integrals versus some $x$-dependent functions but rather as the action of given functionals (defined as the ones appearing in the AE of cyclic integrals) on a particular kind of functions. In this paper, we only discuss the leading order in $x$ part of the action although it is quite clear how to apply the method we present so as to obtain further, sub-leading, corrections. Of course, the size and complexity of the formulae will grow very quickly with the precision of the corrections

The article is organized as follows. In section 2 we remind some properties of the AE of the generalized sine kernel which is a building block of cyclic integrals (1.1). We prove some results on the structure of these asymptotics. In section 3, we recall the link between the generalized sine kernel and cyclic integrals. We also remind the overall properties of their AE and derive some sum-rules. Finally, in section 4, we apply these sum rules so as to determine explicitly the leading $\mathrm{O}(1)$ part of the action of these functionals on a certain class of $x$-dependent functions.

\section{The generalized sine kernel}

The generalized sine kernel is an integral operator $I+V$ on $L^{2}([-q ; q])$. Its kernel $V$ reads

$$
V(\lambda, \mu)=\gamma \sqrt{F(\lambda) F(\mu)} \frac{e(\lambda) / e(\mu)-e(\mu) / e(\lambda)}{2 i \pi(\lambda-\mu)}, \quad e(\lambda)=\mathrm{e}^{i x p(\lambda) / 2+g(\lambda) / 2} .
$$

The asymptotic behavior of the Fredholm determinant of this kernel was derived in [10] under the assumption of $p, F, g$ holomorphic in a neighborhood of $[-q ; q], p$ injective on this neighborhood and $|\gamma|$ small enough. We remind that the $\mathrm{AE}$ of its logarithm has the structure

$$
\ln \operatorname{det}[I+V]=\sum_{N=-1}^{+\infty} \frac{1}{x^{N}} \mathcal{R}^{(N, 0)}[p, g, v]+\sum_{m \in \mathbb{Z}^{*}} \sum_{N \geq 2|m|} \frac{1}{x^{N}} \mathcal{R}^{(N, m)}[p, g, v] \mathrm{e}^{i m x\left(p_{+}-p_{-}\right)} x^{m\left(v_{+}+v_{-}\right)}
$$

Here and in the following, we use the notation $H_{ \pm}=H( \pm q)$ for the boundary values of any function $H$. The function $v$ is related to $F$ by

$$
v(\lambda)=\frac{i}{2 \pi} \ln (1+\gamma F(\lambda)) .
$$

and $\mathcal{R}^{(N, m)}[p, g, v], m \in \mathbb{Z}$, are functional in $p, g$ and $v$. They are also polynomials in $\ln x$ of degree $N$ (we decided not to insist explicitly on this dependence in order to keep the notation light). $\mathcal{R}^{(N)}$ is expressible in terms of integrals over $[-q ; q]$ involving $v$ and products of functions in $p_{ \pm}, g_{ \pm}, v_{ \pm}$or of their higher order derivatives.

Actually, it is easy to see from the details of the recursive construction of $\mathcal{R}^{(N, m)}$ given in [10] that these functionals only produce algebraic expressions in $p$ or its derivatives. Moreover, only $p_{ \pm}^{\prime}$ or $p_{+}-p_{-}$appear in the denominator of such expressions 1 .

Note that one can represent the operator $I+V$ in several ways. Namely, the kernels $V_{p, F, g}$ and $V_{p-i g / x, F, 0}$ parameterize the same generalized sine kerne 2 . However, these two different parameterizations lead to, a priori

\footnotetext{
${ }^{1}$ Another argument to convince oneself of this fact is that, otherwise, the AE would be ill-defined on the functions fulfilling the assumptions of [10]. Indeed, the AE is obtained to any order in $x$ only by assuming holomorphy of the involved functions, smallness of $|\gamma|$ and injectivity of $p$. These conditions can only guarantee that $p_{+}-p_{-}$and $p_{ \pm}^{\prime}$ do not vanish.

${ }^{2}$ Here we have explicitly stressed out the dependence of $V$ on the functions $p, F, g$
} 
distinct, asymptotic series 3 (2.2). In virtue of the uniqueness of an asymptotic expansions, these have to coincide

$$
\begin{aligned}
\sum_{N=-1}^{+\infty} \frac{1}{x^{N}} \mathcal{R}^{(N, 0)}[p, g, v]+\sum_{m \in \mathbb{Z}^{*}} \sum_{N \geq 2|m|} \frac{1}{x^{N}} \mathcal{R}^{(N, m)}[p, g, v] \mathrm{e}^{i m x\left(p_{+}-p_{-}\right)} x^{m\left(v_{+}+v_{-}\right)} \sim \\
\sum_{N=-1}^{+\infty} \frac{1}{x^{N}} \mathcal{R}^{(N, 0)}[p-i g / x, 0, v]+\sum_{m \in \mathbb{Z}^{*}} \sum_{N \geq 2|m|} \frac{1}{x^{N}} \mathcal{R}^{(N, m)}[p-i g / x, 0, v] \mathrm{e}^{i m x\left(p_{+}-p_{-}\right)} \mathrm{e}^{m\left(g_{+}-g_{-}\right)} x^{m\left(v_{+}+v_{-}\right)} .
\end{aligned}
$$

Due to the aforementioned properties of the functionals $\mathcal{R}^{(N, m)}$, the dependence in the RHS of (2.4) is algebraic in $p-i g / x$ and the only terms appear in the denominator have a well defined Taylor series (ie one that only produces corrections in respect to the $x \rightarrow+\infty$ limit). Hence, $\mathcal{R}^{(N)}[p-i g / x, 0, v]$ can be expanded in a Taylor series of inverse powers of $x$. Such an expansion yields $\mathcal{R}^{(N)}[p, 0, v]$ and terms that involve $g$ but are subdominant with respect to it. In other words, the structure in $g$ of the RHS of (2.4) follows by reordering and gathering the powers in $x$ issued from the various Taylor series expansions in the LHS of (2.4). The mechanism of this effect can be seen explicitly on the leading asymptotics of $\ln \operatorname{det}\left[I+V_{p, g, F}\right]$ :

$$
x \mathcal{R}^{(-1,0)}[p, 0, v]=x \int_{-q}^{q} p^{\prime}(\lambda) v(\lambda) \mathrm{d} \lambda
$$

The substitution $p \mapsto p-i g / x$ produces

$$
x \int_{-q}^{q} p^{\prime}(\lambda) v(\lambda) \mathrm{d} \lambda-i \int_{-q}^{q} g^{\prime}(\lambda) v(\lambda) \mathrm{d} \lambda .
$$

This additional $g$-dependent term is precisely the one appearing in the $\mathrm{O}(1)$ asymptotics of $\ln \operatorname{det}\left[I+V_{p, F, g}\right], c f$ [10].

\subsection{Some results on the structure of the asymptotic series}

One can build on the two ways of presenting the AE so as to deduce some properties on the structure of the coefficients involved in the AE (2.2). Such results can hardly by inferred from a direct computation based on the techniques presented in [10]. We prove the results that are important for the purpose of the further analysis in the two lemmas below.

Lemma 2.1 The $1 / x^{N}$ order in the $A E$ of $\ln \operatorname{det}[I+V]$ contains the term

$$
\sum_{\sigma= \pm} \frac{1}{N}\left(\frac{i g_{\sigma}^{\prime}}{p_{\sigma}^{\prime}}\right)^{N} v_{\sigma}^{2}
$$

This is the highest possible power of $g$ that can appear in the non-oscillating part of the $x^{-N}$ order in the AE of ln $\operatorname{det}[I+V](2.2)$.

\footnotetext{
${ }^{3}$ We stress that one can also apply the results of [10] in the case of an $x$-dependent $p$ function, as long as $p=p_{0}+\mathrm{o}(1)$ and $p_{0}$ is holomorphic in a neighborhood of $[-q ; q]$ and injective on this neighborhood. This stems from the fact that when $x \rightarrow+\infty$, only $p_{0}$ plays a role in the details of the analysis carried in [10].
} 
Proof - As it was discussed previously, it is possible to deduce the whole $g$-dependence of $\mathcal{R}^{(N, 0)}[p, g, v]$ by expanding $\mathcal{R}^{(0,0)}[p-i g / x, 0, v], \ldots, x^{1-N} \mathcal{R}^{(N-1,0)}[p-i g / x, 0, v]$ in to Taylor series of inverse powers of $x$. Note that the $g$-dependent part of $x^{-k} \mathcal{R}^{(k, 0)}[p-i g / x, 0, v], k \geq N$ only produces subdominant contribution with respect to $x^{-N} \mathcal{R}^{(N)}$. Moreover, we did not include $\mathcal{R}^{(-1,0)}$ in the list, as we have already seen (2.6) that it only generates the $g$ dependent part of $\mathcal{R}^{(0)}[p, g, v]$.

The Taylor expansion into inverse powers of $g$ of the functional $R^{(\ell, 0)}[p-i g / x, 0, v]$ is of the type

$$
R^{(\ell, 0)}[p, 0, v]+\sum_{n \geq 1} x^{-n} F_{n}^{(\ell)}[p, g, v]
$$

The functional $F_{n}^{(\ell)}[p, g, v]$ is homogeneous in $g$ of order $n$, ie $F_{n}^{(p)}[p, t g, v]=t^{n} F_{n}^{(p)}[p, g, v]$. In other words it only contains powers of $g$ whose total degree in $g$ is $n$, for instance terms of the type

$$
\left(g_{+}\right)^{n_{+}^{(0)}} \ldots\left(g_{+}^{(k)}\right)^{n_{+}^{(k)}}\left(g_{-}\right)^{n_{-}^{(0)}} \ldots\left(g_{-}^{(k)}\right)^{n_{-}^{(k)}}, \quad \text { where } \sum_{p=1}^{k} n_{+}^{(p)}+n_{-}^{(p)}=n
$$

Hence, the highest possible degree in $g$ of $\mathcal{R}^{(N, 0)}[p, g, v]$ can only be issued from the $N^{\text {th }}$ term of the Taylor series in $x^{-1}$ of $\mathcal{R}^{(0)}[p-i g / x, 0, v]$. As $-\Sigma_{\sigma= \pm} v_{\sigma}^{2} \ln p_{\sigma}^{\prime}$ represents the $p$ dependent part of $\mathcal{R}^{(0)}[p, 0, v]$, the substitution $p \mapsto p-i g / x$ generates the series of inverse powers of $x$ :

$$
-\sum_{\sigma= \pm} v_{\sigma}^{2} \ln p_{\sigma}^{\prime}+\sum_{\sigma= \pm} v_{\sigma}^{2} \sum_{\ell \geq 1} \frac{1}{\ell x^{\ell}}\left(\frac{i g_{\sigma}^{\prime}}{p_{\sigma}^{\prime}}\right)^{\ell}
$$

Thus, the highest possible degree in $g$ contained in $\mathcal{R}^{(N, 0)}[p, g, v]$ is $N$ and the corresponding contribution is $N^{-1} \cdot\left[\left(i g_{+}^{\prime} / p_{+}^{\prime}\right)^{N}+\left(i g_{-}^{\prime} / p_{-}^{\prime}\right)^{N}\right]$.

One can also prove an analogous lemma in respect to the oscillating part.

Lemma 2.2 The $1 / x^{N+2}$ part of the oscillating corrections with period $\mathrm{e}^{i \sigma x\left(p_{+}-p_{-}\right)}, \sigma= \pm$, contains the series

$$
\mathrm{e}^{\sigma\left(g_{+}-g_{-}\right)} \sum_{k=0}^{n}\left(\frac{i g_{+}^{\prime}}{p_{+}^{\prime}}\right)^{k}\left(\frac{i g_{-}^{\prime}}{p_{-}^{\prime}}\right)^{n-k} \widetilde{O}_{n, k}[\sigma v]
$$

We have introduced the functional

$$
\begin{aligned}
\widetilde{O}_{n, k}[v] & =\frac{[2 q x]^{2\left(v_{+}+v_{-}\right)}}{(2 q)^{2}} p_{+}^{\prime 2 v_{+}-1} p_{-}^{\prime 2 v_{-}-1} \frac{\Gamma\left(1-v_{-}\right) \Gamma\left(1-v_{+}\right) \kappa_{-}^{2}}{\Gamma\left(v_{-}\right) \Gamma\left(v_{+}\right) \kappa_{+}^{2}} \frac{\left(2 v_{+}-1\right)_{k}\left(2 v_{-}-1\right)_{n-k}}{k !(n-k) !}, \\
\text { with } \ln \kappa(\lambda) & =\int_{-q}^{q} \frac{v(\lambda)-v(\mu)}{\lambda-\mu} \mathrm{d} \mu .
\end{aligned}
$$

Proof - The first oscillating term appearing in the asymptotic expansion of $\ln \operatorname{det}[I+V]_{\mid g=0}$ reads [10]:

$$
\frac{1}{x^{2}} \sum_{\sigma= \pm} \widetilde{O}_{0,0}[\sigma v] \mathrm{e}^{i x \sigma\left(p_{+}-p_{-}\right)} \quad \text { so that } \quad R^{(2, \sigma)}[p, g, v]=x^{-2 \sigma\left(v_{+}+v_{-}\right)} \widetilde{O}_{0,0}[\sigma v]
$$

Just as for the non-oscillating corrections discussed above, it is the only one responsible for producing the highest degree in $g$ of the oscillating corrections subordinate to the frequency $\mathrm{e}^{i x \sigma\left(p_{+}-p_{-}\right)}, \sigma= \pm$. To deduce the structure 
of these terms, we set $p \mapsto p-i g / x$ in the above functional and perform a Taylor series expansion. For $x$ large enough:

$$
\frac{1}{\left(1-\epsilon_{1} / x\right)^{\alpha}\left(1-\epsilon_{2} / x\right)^{\beta}}=\sum_{n \geq 0} \frac{1}{x^{n}} \sum_{k=0}^{n} \epsilon_{2}^{n-k} \epsilon_{1}^{k} \frac{(\alpha)_{k}(\beta)_{n-k}}{k !(n-k) !}, \quad \text { where } \quad(\alpha)_{k}=\frac{\Gamma(\alpha+k)}{\Gamma(\alpha)} .
$$

We get that the highest degree in $g$ appearing in the $x^{-2-N}$ part of the oscillating $\mathrm{e}^{i \sigma\left(p_{+}-p_{-}\right)}$corrections is given by

$$
\sum_{\sigma= \pm} \sum_{k=0}^{N} \widetilde{O}_{N, k}[\sigma v] \mathrm{e}^{\sigma\left(g_{+}-g_{-}\right)}\left(\frac{i g_{+}^{\prime}}{p_{+}^{\prime}}\right)^{k}\left(\frac{i g_{-}^{\prime}}{p_{-}^{\prime}}\right)^{N-k}
$$

It is clear that one can look at lower order terms like $\mathcal{R}^{(1,0)}[p, 0, v]$ so as to obtain the coefficient in front of the terms of total degree $N-1$ in $g$ at any order in $x^{-N}$. In the next sections, we will actually need the highest degree in $g$ dependence in the AE of

$$
J_{n}[p, g, F]=\frac{(-1)^{n-1}}{(n-1) !} \partial_{\gamma}^{n} \ln \operatorname{det}[I+V]_{\mid \gamma=0} .
$$

The latter is easily deduced from the previous lemmas due to the fact that the $\mathrm{AE}$ of $\ln \operatorname{det}[I+V]$ in uniform to any finite order derivative in $\gamma$. We gather these results in the

Corollary 2.1 The highest degree of $g$ in the non-oscillating $x^{-N}$ order of the asymptotic expansion of $J_{n}[p, g, F]$ reads

$$
\frac{(-1)^{n-1}}{(n-1) !} \sum_{\sigma= \pm} \frac{1}{N}\left(\frac{i g_{\sigma}^{\prime}}{p_{\sigma}^{\prime}}\right)^{N}\left(F_{\sigma}\right)^{n} \partial_{\gamma}^{n}\left(v_{0}^{2}\right)_{\mid \gamma=0} \quad \text { where } \quad v_{0}=\frac{i}{2 \pi} \ln (1+\gamma) .
$$

Similarly, the highest degree in $g$ present in the $x^{-N-2}$ oscillating part at frequency $\mathrm{e}^{i x \sigma\left(p_{+}-p_{-}\right)}, \sigma= \pm$, in the AE of $J_{n}[p, g, F]$ is given by the series

$$
\frac{(-1)^{n-1}}{(n-1) !} \mathrm{e}^{\sigma\left(g_{+}-g_{-}\right)} \sum_{k=0}^{N}\left(\frac{i g_{+}^{\prime}}{p_{+}^{\prime}}\right)^{k}\left(\frac{i g_{-}^{\prime}}{p_{-}^{\prime}}\right)^{N-k} \partial_{\gamma}^{n} \widetilde{O}_{N, k}[\sigma v]_{\gamma=0} .
$$

\section{Cyclic multiple integrals}

One of the main results of [10], was the proof of the asymptotic expansion for cyclic integrals:

$$
\mathcal{I}_{n}\left[\mathcal{F}_{n}\right]=\int_{\Gamma([-q ; q])} \frac{\mathrm{d}^{n} z}{(2 i \pi)^{n}} \int_{-q}^{q} \frac{\mathrm{d}^{n} \lambda}{(2 i \pi)^{n}} \mathcal{F}_{n}\left(\begin{array}{c}
\{\lambda\} \\
\{z\}
\end{array}\right) \prod_{k=1}^{n} \frac{\mathrm{e}^{i x\left[p\left(z_{k}\right)-p\left(\lambda_{k}\right)\right]}}{\left(\lambda_{k}-z_{k}\right)\left(\lambda_{k}-z_{k+1}\right)} .
$$

There, $\mathcal{F}_{n}$ is a holomorphic function of the $n$ variables $\{z\}$ and the $n$ variables $\{\lambda\}$ that is symmetric separately in respect to variables belonging to each of these two sets. $\Gamma([-q ; q])$ is a sufficiently small loop of index 1 around $[-q ; q]$. Lastly, we agree upon $z_{n+1} \equiv z_{1}$. It was shown in [10], that there exists functionals $I_{n}^{(N, m)}$ such that the asymptotic expansion of $\mathcal{I}_{n}\left[\mathcal{F}_{n}\right]$ reads

$$
\mathcal{I}_{n}\left[\mathcal{F}_{n}\right]=\sum_{m \in \mathbb{Z}} \sum_{N \geq 2|m|-\delta_{m, 0}} \mathrm{e}^{i m x\left(p_{+}-p_{-}\right)} \frac{1}{x^{N}} I_{n}^{(N, m)}\left[\mathcal{F}_{n}\right]
$$


This equality is to be understood in the sense of an asymptotic expansion. In particular, it excludes any $\mathrm{O}\left(x^{-\infty}\right)$ terms. For the purpose of our study, we now recall a couple of general properties of the functionals appearing in (3.2). First, all the oscillating terms $\mathrm{e}^{i m x\left(p_{+}-p_{-}\right)}$are factored out from $I_{n}^{(N, m)}$ and these functionals are polynomial in $\ln x$ of at most degree $n+N$. Their action involves the finite-difference operator

$$
\delta_{z_{\ell}}^{(t)}(\mu) \cdot \mathcal{G}_{n}\left[\left\{\left\{z_{i}\right\}^{\bar{p}_{i}}\right\}_{i=1, \ldots, r}\right]=\mathcal{G}_{n}\left[\left\{\left\{z_{i}\right\}^{\bar{p}_{i}}\right\}_{i=1, \ldots, r}\right]-\mathcal{G}_{n}\left[\left\{\left\{z_{k}\right\}^{\bar{p}_{k}}\right\}_{k \neq \ell},\{\mu\}^{t},\left\{z_{\ell}\right\}^{\bar{p}_{\ell}-m}\right] .
$$

The function $\mathcal{G}_{n}$ appearing in (3.3) is assumed symmetric in its variables. Moreover the notation, $\left\{z_{k}\right\} \bar{p}_{k}$ means that the variable $z_{k}$ is repeated $\bar{p}_{k}$ times

$$
\left\{z_{k}\right\}^{\bar{p}_{k}}=(\underbrace{z_{1}, \ldots, z_{1}}_{\bar{p}_{1} \text { times }}, \ldots, \underbrace{z_{r}, \ldots z_{r}}_{\bar{p}_{r}}) .
$$

Hence, $\partial_{z \ell}^{(t)}(\mu)$ acts by replacing $t \leq \bar{p}_{\ell}$ variables $z_{\ell}$ by $\mu$ and then performing the difference with the function one started with. The action of $I_{n}^{(N, m)}$ takes the form

$$
I_{n}^{(N, m)}\left[\mathcal{F}_{n}\right]=\Im_{N}^{(m)} * \sum_{\Sigma k_{\ell} \leq N-2|m|} G_{\left\{k_{\ell}\right\}}^{\Im_{N}^{(m)}} \cdot\left\{\partial_{z_{1}}^{k_{1}} \ldots \partial_{z_{r}}^{k_{r}} \prod_{\ell=1}^{r} \prod_{t=1}^{n} \prod_{j=1}^{p_{\ell, t}}\left\{\int_{-q}^{q} \frac{\mathrm{d} \mu_{\ell, t, j}}{z_{\ell}-\mu_{\ell, t, j}} \delta_{z \ell}^{(t)}\left(\mu_{\ell, t, j}\right)\right\} \cdot \mathcal{F}_{n}\left(\begin{array}{c}
\left\{z_{\ell}\right\}^{\bar{p}_{\ell}} \\
\left\{z_{\ell}\right\}^{\bar{p}_{\ell}+\epsilon_{\ell}}
\end{array}\right)\right\}_{z_{\ell}=\sigma_{\ell q}}
$$

Here $\mathfrak{S}_{N}^{(m)} *$ is a shorthand notation for the multiple summation symbol

$$
\mathfrak{S}_{N}^{(m)} *=\sum_{r=1+|m|}^{n} \sum_{\sigma_{\ell}= \pm} \sum_{\substack{\epsilon_{\ell}= \pm 1,0 \\ \Sigma \epsilon_{\ell}=0}} \sum_{\substack{\Sigma \bar{p}_{\ell}=n \\ p_{\ell, 0} \geq 0}}
$$

over the points $\sigma_{\ell} q=, \sigma_{\ell}= \pm$, where the variables $z_{\ell}$ are evaluated, the integers $\epsilon_{\ell}= \pm 1,0, \ell=1, \ldots r$ parameterizing the sets of unequal variables and integers $p_{\ell, t}$ labeling the number of finite difference operators involved. These integers are subject to the constraints $p_{\ell, t} \geq 0, p_{\ell, 0} \geq 1$ and $\bar{p}_{\ell}=\Sigma_{t=1}^{n} t p_{\ell, t}, \quad \Sigma_{\ell=1}^{r} \epsilon_{\ell}=0$ and $\Sigma_{\ell=1}^{r} \epsilon_{\ell} p_{\sigma_{\ell}}=m\left(p_{+}-p_{-}\right)$. The coefficients $G_{\left\{k_{\ell}\right\}}^{\Xi_{N}^{(m)}}$ depend on all of these additional summation indices and are polynomials in $\ln x$ of degree $n+N$. We chose to keep this dependence implicit, so as to have as light notations as possible.

$I_{n}^{(N ; m)}$ is thus written as a sum of terms. Each of these terms corresponds to several operation performed on $\mathcal{F}_{n}$. The set the initial $\lambda$-variables of $\mathcal{F}_{n}$ is set to $\left\{z_{\ell}\right\}^{\bar{p}_{\ell}}$ and the $z$-variables to $\left\{z_{\ell}\right\}^{\bar{p}_{\ell}+\epsilon_{\ell}}$. It thus follows that most of the variables in the upper and lower hypergeometric type notation for $\mathcal{F}_{n}$ are set equal. The variables $\{z \ell\}$ are acted upon by the finite-difference operators, the result of the action being integrated versus a $z_{\ell}$ dependent weight along $[-q ; q]$. After the action of the finite-difference operators, one acts with the derivative operators $\partial_{z_{1}}^{k_{1}} \ldots \partial_{z_{r}}^{k_{r}}$ with $0 \leq \Sigma_{s=1}^{r} k_{s} \leq N-2|m|$ and sets $z_{\ell}=\sigma_{\ell} q, \sigma_{\ell}= \pm$. One can be more specific about the structure of the part of $I_{n}^{(N, m)}$ containing only the highest order $N-2|m|$ of derivatives. There, the variables $z_{1}, \ldots, z_{r}$ are set equal to $\sigma_{s} q=z_{s}$, where the sequence $\sigma_{s} \in\{ \pm 1\}$ and has precisely $|m|$ jumps. For instance, when $m=0$, only two sequences $\sigma_{\ell}$ are possible, $\sigma_{s}=\sigma \forall s, \sigma= \pm$.

There exist a relation between the Fredholm determinant ln det $[I+V]$ and cyclic integrals involving a special class of of symmetric functions in $n$ variables $\{\lambda\}$ and $n$ variables $\{z\}$ that we call pure product functions:

$$
\mathcal{F}_{n}^{(\varphi, g)}\left(\begin{array}{c}
\{\lambda\} \\
\{z\}
\end{array}\right)=\prod_{k=1}^{n} \varphi\left(\lambda_{k}\right) \mathrm{e}^{g\left(z_{k}\right)}
$$


Indeed, on the one hand by looking at the $n^{\text {th }}$ term in the series in $\gamma$ of $\ln \operatorname{det}[I+V]$ and, on the other hand, by computing the residues at $z_{k}=\lambda_{k}$ or $z_{k}=\lambda_{k+1}$ in (1.1), one shows that

$$
J_{n}\left[p, g, \varphi \mathrm{e}^{g}\right]=\mathcal{I}_{n}\left[\mathcal{F}_{n}^{(\varphi, g)}\right] .
$$

This observation was used in [10] to build on the AE of $\ln \operatorname{det}[I+V]$ so as to deduce the one of general cyclic integrals (1.1). Due to (2.2) and (3.8) the $g$-dependent part of the AE of $\mathcal{I}_{n}\left[\mathcal{F}_{n}^{(\varphi, g)}\right]$ can be deduced from the one of $\mathcal{R}^{(N, m)}(x)\left[p, g, v^{\left(\varphi \mathrm{e}^{g}\right)}\right]$, where $v^{\left(\varphi \mathrm{e}^{g}\right)}$ is obtained from $v$ by setting $F=\varphi \mathrm{e}^{g}$ in (2.3). Yet, this $g$-dependence has also to be reproduced if one chooses to act with the functionals $I_{n}^{(N, m)}(3.2)$ on $\mathcal{F}_{n}^{(\varphi, g)}$ instead. This fact allows us to derive certain sum-rules for part of the terms appearing in (3.5). Such identities will be used in the next section so as to obtain the leading $\mathrm{O}(1)$ order in $x$ of the action of $x^{-N} I_{n}^{(N, m)}$ on $x$-dependent functions.

\subsection{Non-oscillating sum-rules}

We identify the part of $I_{n}^{(N, 0)}\left[\mathcal{F}_{n}^{(\varphi, g)}\right]$ producing the highest order in $g$ of $J_{n}\left[p, g, \varphi \mathrm{e}^{g}\right]$. Due to the pure-product structure of $\mathcal{F}_{n}^{(\varphi, g)}$ we have

$$
\mathcal{F}_{n}^{(\varphi, g)}\left(\begin{array}{c}
\left\{z_{\ell}\right\}^{\bar{p}_{\ell}} \\
\left\{z_{\ell}\right\}^{\bar{p}_{\ell}+\epsilon_{\ell}}
\end{array}\right)=\prod_{s=1}^{r} F\left(z_{s}\right)^{\bar{p}_{s}} \mathrm{e}^{\epsilon_{s} g\left(z_{s}\right)}, \quad F=\varphi \mathrm{e}^{g} .
$$

This function admits a simple representation for the action of $\mathrm{\partial}_{z}^{(t)}\left(\mu_{\ell, t, j}\right)$ :

$$
\begin{aligned}
\prod_{\ell=1}^{r} \prod_{t=1}^{n} \prod_{j=1}^{p_{\ell, t}}\left\{\int_{-q}^{q} \frac{\mathrm{d} \mu_{\ell, t, j}}{z_{\ell}-\mu_{\ell, t, j}} \delta_{z \ell}^{(t)}\left(\mu_{\ell, t, j}\right)\right\} & \prod_{s=1}^{r}\left\{F\left(z_{s}\right)^{\bar{p}_{s}} \mathrm{e}^{\epsilon_{s} g\left(z_{s}\right)}\right\}= \\
& \prod_{s=1}^{r}\left\{F\left(z_{s}\right)^{p_{s, 0}} \mathrm{e}^{\epsilon_{s} g\left(z_{s}\right)} \prod_{t=1}^{n}\left[\int_{-q}^{q} \frac{\left[F\left(z_{s}\right)\right]^{t}-[F(\mu)]^{t}}{z_{s}-\mu} \mathrm{d} \mu\right]^{p_{s t}}\right\} .
\end{aligned}
$$

Thence,

$$
I_{n}^{(N, 0)}\left[\mathcal{F}_{n}\right]=\mathfrak{S}_{N}^{(0)} * \sum_{\Sigma k_{\ell} \leq N} G_{\left\{k_{\ell}\right\}}^{\mathfrak{S}_{N}^{(0)}}\left\{\partial_{z_{1}}^{k_{1}} \ldots \partial_{z_{r}}^{k_{r}} \prod_{s=1}^{r}\left\{F\left(z_{s}\right)^{p_{s, 0}} \mathrm{e}^{\epsilon_{s} g\left(z_{s}\right)}\right\} \prod_{t=1}^{n}\left\{\int_{-q}^{q} \frac{\left[F\left(z_{s}\right)\right]^{t}-[F(\mu)]^{t}}{z_{s}-\mu} \mathrm{d} \mu\right\}^{p_{s t}}\right\}
$$

The maximal degree in $g$ can only stem from the part of the sum involving the highest possible number of derivatives. Indeed, it is obtained by hitting with all of the derivatives $\partial_{z_{1}}^{k_{1}} \ldots \partial_{z_{r}}^{k_{r}}$ on the exponent $\prod_{k=1}^{r} \mathrm{e}^{\epsilon_{k} g\left(z_{k}\right)}$. Any other type of action produces a lower degree in $g$. More explicitly, for an holomorphic function $H$

$$
\partial_{z_{1}}^{k_{1}} \ldots \partial_{z_{r}}^{k_{r}}\left\{\prod_{s=1}^{r} \mathrm{e}^{\epsilon_{s} g\left(z_{s}\right)} \cdot H(\{z\})\right\}=\prod_{s=1}^{r}\left(\epsilon_{s} g^{\prime}\left(z_{s}\right)\right)^{k_{s}} \mathrm{e}^{\epsilon_{s} g\left(z_{s}\right)} \cdot H(\{z\})+\mathrm{LD}(g)
$$

$\mathrm{LD}(g)$ stands for the terms that are of a lower degree in $g$. Once the derivatives have been acted with, one should set $z_{1}=\cdots=z_{r}=\sigma q$ as there are no jumps in the sequence $\sigma_{\ell}$ when $m=0$ and $\Sigma k_{\ell}=N$. In particular, this allows us to simplify the products of the exponents

$$
\prod_{s=1}^{r} \mathrm{e}^{\epsilon_{s} g(\sigma q)}=\exp \left\{g(\sigma q) \sum_{s=1}^{r} \epsilon_{s}\right\}=1 \quad \text { due to the constraint } \quad \sum_{s=1}^{r} \epsilon_{s}=0 .
$$


By lemma 2.1, the highest possible degree in $g$ appearing in the non-oscillating $x^{-N}$ order of the AE of $\ln \operatorname{det}[I+V]$ is given by (2.7). Thus we obtain the sum-rule:

$$
\begin{array}{r}
I_{n}^{(N, 0)}\left[\mathcal{F}_{n}^{(\varphi, g)}\right]=\mathbb{S}_{N}^{(0)} * \sum_{\Sigma k_{\ell}=N} G_{\left\{k_{\ell}\right\}}^{\Xi_{s=1}^{(0)}} \prod_{s=1}^{r}\left\{\left[\epsilon_{s} g^{\prime}\left(z_{s}\right)\right]^{k_{s}}\left[F\left(z_{s}\right)\right]^{p_{s, 0}} \prod_{t=1}^{n}\left\{\int_{-q}^{q} \frac{\left[F\left(z_{s}\right)\right]^{t}-[F(\mu)]^{t}}{z_{s}-\mu} \mathrm{d} \mu\right\}^{p_{s t}}\right\} \\
=\sum_{\sigma= \pm} \frac{1}{N}\left(\frac{i g_{\sigma}^{\prime}}{p_{\sigma}^{\prime}}\right)^{N}\left\{\frac{(-1)^{n-1}}{(n-1) !} \partial_{\gamma}^{n}\left(v_{0}\right)_{\mid \gamma=0}\right\} F_{\sigma}^{n} .
\end{array}
$$

\subsection{Oscillating sum-rules}

One can repeat word for word the above analysis in the case of the action of $I_{n}^{(N, m)}, m= \pm$, on pure product functions. One gets that

$$
\begin{gathered}
\mathfrak{S}_{N}^{(m)} * \sum_{\Sigma k_{\ell}=N-2} G_{\left\{k_{\ell}\right\}}^{\mathfrak{S}_{N}^{(m)}} \prod_{s=1}^{r}\left\{\left[\epsilon_{s} g^{\prime}\left(z_{s}\right)\right]^{k_{s}}\left[F\left(z_{s}\right)\right]^{p_{s, 0}} \prod_{t=1}^{n}\left\{\int_{-q}^{q} \frac{\left[F\left(z_{s}\right)\right]^{t}-[F(\mu)]^{t}}{z_{s}-\mu} \mathrm{d} \mu\right\}^{p_{s t}}\right\} \\
=\sum_{k=0}^{N-2}\left(\frac{i g_{+}^{\prime}}{p_{+}^{\prime}}\right)^{k}\left(\frac{i g_{-}^{\prime}}{p_{-}^{\prime}}\right)^{N-k-2} \partial_{\gamma}^{n} \widetilde{O}_{N, k}[\sigma v]_{\mid \gamma=0} .
\end{gathered}
$$

The only difference in the proof of the oscillating sum-rule lies in the specialization of the variables $z_{\ell}$ to $\sigma_{\ell} q$. For a maximal number $N-2$ of derivatives in $I_{n}^{(N, m)}$ there can be only one jump in the sequence $\sigma_{\ell}(3.6)$. Moreover, the sequence $\epsilon_{s}$ has to fulfill $\Sigma \epsilon_{s} p_{\sigma_{s}}=m\left(p_{+}-p_{-}\right)$. It thus follows that $\Sigma \epsilon_{s} p_{\sigma_{s}}=m\left(g_{+}-g_{-}\right)$and the resulting prefactor cancels with the one appearing (2.11).

\section{The action on $x$-dependent functions}

We will now apply the sum-rules derived in the previous section so as to obtain the $\mathrm{O}(1)$ part of the action of the functionals $I_{n}^{(N ; m)}, m \in\{0, \pm 1\}$, on a certain class of $x$-dependent functions. We however first define the class of functions that we consider in the following.

\subsection{The type of $x$-dependent function}

In the rest of this article, we assume that $\mathcal{F}_{n}$ has the typical structure of functions involved in the asymptotic analysis of correlation functions of integrable models [9]. Such functions $\mathcal{F}_{n}$ are written as

$$
\mathcal{F}_{n}\left(\begin{array}{c}
\{\lambda\} \\
\{z\}
\end{array}\right)=\exp \left\{x \mathcal{G}_{n}\left(\begin{array}{c}
\{\lambda\} \\
\{z\}
\end{array}\right)+\ln x \mathcal{H}_{n}\left(\begin{array}{c}
\{\lambda\} \\
\{z\}
\end{array}\right)\right\} \mathcal{W}_{n}\left(\begin{array}{c}
\{\lambda\} \\
\{z\}
\end{array}\right) \prod_{i=1}^{n} \mathcal{V}_{n}\left(\lambda_{i} \mid \begin{array}{l}
\{\lambda\} \\
\{z\}
\end{array}\right) .
$$

All of the function in (4.1) are symmetric in the $\{\lambda\}$ and $\{z\}$ variables. Moreover, they satisfy reduction properties in respect to variables appearing in hypergeometric notation, eg:

$$
\mathcal{W}_{n}\left(\begin{array}{l}
\{\lambda\}_{1}^{n} \\
\{z\}_{1}^{n}
\end{array}\right)_{\left.\right|_{z s}=\lambda_{k}}=\mathcal{W}_{n}\left(\begin{array}{c}
\{\lambda\}_{1, \neq k}^{n} \\
\{z\}_{1, \neq s}^{n}
\end{array}\right) .
$$

In the above equation, we have indicated the label dependence of the variables so as to make clear what we mean by reduction property. Yet, as long as it does not lead to confusion, we will omit to write this dependence explicitly later on. 


\subsection{The action of $I_{n}^{(N, 0)}$}

Proposition 4.1 Let $\mathcal{F}_{n}$ be as in (4.1), then

$$
x^{-N} I_{n}^{(N ; 0)}\left[\mathcal{F}_{n}\right]=\mathrm{e}^{x \mathcal{G}+\ln x \mathcal{H}} W\left\{\frac{(-1)^{n-1}}{(n-1) !} \partial_{\gamma}^{n}\left(v_{0}\right)_{\mid \gamma=0}\right\} \sum_{\sigma= \pm} \frac{1}{N}\left(\frac{i \mathcal{G}^{\prime}(\sigma q)}{p^{\prime}(\sigma q)}\right)^{N} \mathcal{V}^{N}(\sigma q)+\mathrm{o}(1) .
$$

We have introduced shorthand notations for the fully reduced functions

$$
\begin{aligned}
\mathcal{G}=\mathcal{G}_{0}\left(\begin{array}{l}
\cdot \\
\cdot
\end{array}\right) & =\mathcal{G}_{1}\left(\begin{array}{l}
z \\
z
\end{array}\right), \quad \mathcal{H}=\mathcal{H}_{0}\left(\begin{array}{c}
\cdot \\
\cdot
\end{array}\right), \quad \mathcal{W}=\mathcal{W}_{0}\left(\begin{array}{l}
\cdot \\
\cdot
\end{array}\right), \\
\mathcal{V}(\lambda) & =\mathcal{V}\left(\lambda \mid \cdot \begin{array}{l}
\cdot \\
\cdot
\end{array}\right), \quad \mathcal{G}^{\prime}(\lambda)=\partial_{\epsilon} \mathcal{G}_{1}\left(\begin{array}{c}
\lambda \\
\lambda+\epsilon
\end{array}\right)_{\mid \epsilon=0} .
\end{aligned}
$$

Proof -

The key observation is that for functions satisfying reduction properties, may terms in the action of $I_{n}^{(N, m)}$ decouple and one is almost reduced to the pure-product case. Due to the reduction properties of $\mathcal{F}_{n}$, the action of the finite-difference operators simplifies:

$$
\begin{array}{r}
\delta_{z}^{(t)}\left(\mu_{\ell, t, j}\right) \cdot \mathcal{F}_{n}\left(\begin{array}{c}
\left\{z_{s} \bar{p}_{s}\right. \\
\left\{z_{s}\right\}^{\bar{p}_{s}+\epsilon_{s}}
\end{array}\right)=\mathcal{F}_{n-t}\left(\begin{array}{c}
\left\{z_{s} \bar{p}_{s}-t \delta_{s, \ell}\right. \\
\left\{z_{s}\right\}^{\bar{s}_{s}+\epsilon_{s}-t \delta_{s, \ell}}
\end{array}\right)\left\{\left[\mathcal{V}_{n-t}\right]^{t}\left(\begin{array}{l}
\{\ell \\
\left\{z_{s}\right\}^{\bar{s}_{s}-t \delta_{s, \ell}} \\
\left\{z_{s}\right\}_{s}+\epsilon_{s}-t \delta_{s, \ell}
\end{array}\right)\right. \\
\left.-\left[\mathcal{V}_{n-t}\right]^{t}\left(\mu_{\ell, m, j} \mid \begin{array}{c}
\left\{z_{s}\right\}^{\bar{p}_{s}-t \delta_{s, \ell}} \\
\left\{z_{s}\right\}_{s}+\epsilon_{s}-t \delta_{s, \ell}
\end{array}\right)\right\} .
\end{array}
$$

There, $\delta_{s, \ell}$ is the Kronecker $\delta$ symbol. Thus,

$$
\begin{aligned}
& \prod_{\ell=1}^{r} \prod_{t=1}^{n} \prod_{j=1}^{p_{\ell, t}}\left\{\int_{-q}^{q} \frac{\mathrm{d} \mu_{\ell, t, j}}{z_{\ell}-\mu_{\ell, t, j}} \mathrm{~J}_{z}^{(t)}\left(\mu_{\ell, t, j}\right)\right\} \cdot \mathcal{F}_{n}\left(\begin{array}{c}
\left\{z_{s}\right\}^{\bar{p}_{s}} \\
\left\{z_{s}\right\}^{\bar{p}_{s}}+\epsilon_{s}
\end{array}\right)
\end{aligned}
$$

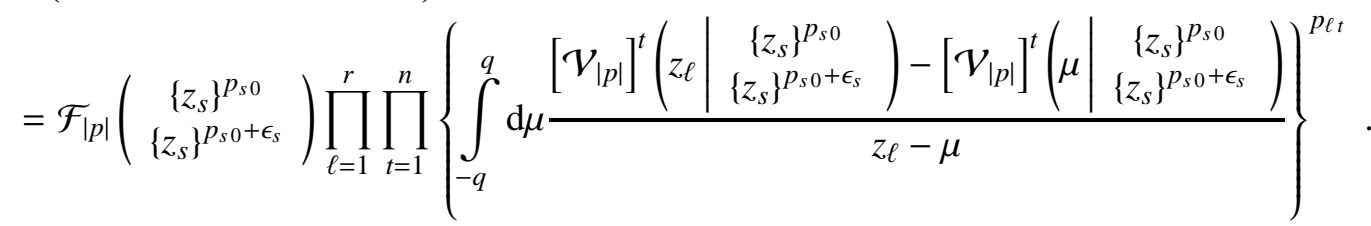

$|p|=\Sigma_{\ell} p_{\ell 0}$ is the number of variables remaining after computing the action the finite-difference operators. Indeed, all the dependence on the variables $\left\{z_{s}\right\}$ that are repeated $p_{s, \ell}$ times, $\ell=1, \ldots, n$, disappears from the function. To evaluate the action of $I_{n}^{(N, 0)}$, one should hit with the product of $z_{s}$ derivatives on the RHS of (4.7) just as it was done in (3.12). Clearly, such an operation generates positive powers of $x$ (by differentiating the exponent $\mathrm{e}^{x \mathcal{G}_{n}}$ ) or of $\ln x$ (by differentiating $\mathrm{e}^{\ln x \mathcal{H}_{n}}$ ). In order to obtain the highest possible power of $x$, the derivative should only hit on the $x \mathcal{G}_{n}$ part of the exponent. In this way $\partial_{z_{1}}^{k_{1}} \ldots \partial_{z_{r}}^{k_{r}}$ produces a contribution of order $x^{k_{1}+\cdots+k_{r}}$. Hence, the largest possible power of $x$ comes from derivatives such that $\Sigma k_{\ell}=N$, and then

$$
\partial_{z_{1}}^{k_{1}} \ldots \partial_{z_{r}}^{k_{r}} \mathcal{F}_{|p|}\left(\begin{array}{c}
\left\{z_{s}\right\}^{p_{s} 0} \\
\left\{z_{s}\right\}^{p_{s 0}+\epsilon_{s}}
\end{array}\right)=x^{N} \prod_{s=1}^{r}\left(\partial_{z_{k}} \mathcal{G}_{|p|}\left(\begin{array}{c}
\left\{z_{s}\right\}^{p_{s 0}} \\
\left\{z_{s}\right\}^{p_{s 0}+\epsilon_{s}}
\end{array}\right)\right)^{k_{s}} \mathcal{F}_{|p|}\left(\begin{array}{c}
\left\{z_{s}\right\}^{p_{s 0}} \\
\left\{z_{s}\right\}^{p_{s 0}+\epsilon_{s}}
\end{array}\right)+\mathrm{O}\left(\frac{x^{N} \ln x}{x}\right)
$$

Note that the positive power $x^{N}$ simplifies with the prefactor in front of $I_{n}^{(N, m)}$. Once the derivatives are computed, one should send all of the z's to $\sigma q, \sigma= \pm$. Hence, due to the conditions $\Sigma \epsilon_{\ell}=0$ the upper and the lower variables 
in hypergeometric notation will be equal. This allows one to apply the reduction properties to $\mathcal{F}_{|p|}$, as well as to most of the variables appearing in $\partial_{z_{k}} \mathcal{G}_{|p|}$. In particular, the reduction properties imply that

$$
\partial_{z_{k}} \mathcal{G}_{|p|}\left(\begin{array}{c}
\left\{z_{s}\right\}^{p_{s 0}} \\
\left\{z_{s}\right\}^{p_{s 0}+\epsilon_{s}}
\end{array}\right)=\epsilon_{k} \partial_{\epsilon} \mathcal{G}_{|p|}\left(\begin{array}{c}
\left\{z_{s}\right\}_{s \neq k}^{p_{s 0}} ; z_{k} \\
\left\{z_{s}\right\}_{s \neq k}^{p_{s 0}+\epsilon_{s}} ; z_{k}+\epsilon
\end{array}\right)_{\mid \epsilon=0} .
$$

Hence, after implementing most of the operations, it follows that, up to o (1) corrections, $\mathcal{W} \mathrm{e}^{x \mathcal{G}+\ln x \mathcal{H}}$ can be pulled out from the sum as it is a constant, and

$$
\begin{aligned}
x^{-N} I_{n}^{(N, 0)}\left[\mathcal{F}_{n}\right]=\mathcal{W} \mathrm{e}^{x \mathcal{G}+\ln x \mathcal{H}} \mathbb{S}_{N}^{(m)} * \sum_{\Sigma k_{\ell}=N} G_{\left\{k_{\ell}\right\}}^{\mathfrak{S}_{N}^{(m)}} & \\
& \times\left\{\prod_{s=1}^{r}\left[\epsilon_{s} \mathcal{G}^{\prime}\left(z_{s}\right)\right]^{k_{s}}\left[\mathcal{V}\left(z_{s}\right)\right]^{p_{s 0}} \prod_{t=1}^{n}\left\{\int_{-q}^{q} \mathrm{~d} \mu \frac{\left[\mathcal{V}\left(z_{s}\right)\right]^{t}-[\mathcal{V}(\mu)]^{t}}{z_{s}-\mu}\right\}\right\}+\mathrm{o}(1)
\end{aligned}
$$

Thus by applying the sum-rule (3.14) we get the claim.

\subsection{The action of $I_{n}^{(N, \pm 1)}$}

We now focus on the action of the functional $x^{-N} I_{n}^{(N, m)}, m= \pm$ and $N \geq 2$, on the class of $x$-dependent functions as in (4.1).

Proposition 4.2 Let $\mathcal{F}_{n}$ be as in (4.1), then

$$
\begin{array}{r}
\frac{I_{n}^{(N ; m)}\left[\mathcal{F}_{n}\right]}{x^{N}}=x^{-2} \mathcal{W}^{(m)} \mathrm{e}^{x \mathcal{G}^{(m)}+\ln x \mathcal{H}^{(m)}} \sum_{k=0}^{N}\left(\frac{i\left(\mathcal{G}^{(m)}\right)_{+}^{\prime}}{p_{+}^{\prime}}\right)^{k}\left(\frac{i\left(\mathcal{G}^{(m)}\right)_{-}^{\prime}}{p_{-}^{\prime}}\right)^{N-k} \partial_{\gamma}^{n}{\widetilde{O_{N}}}_{k}\left[\sigma v^{(m)}\right]_{\mid \gamma=0}+\mathrm{o}\left(x^{-2}\right) \\
\text { with } \quad v^{(m)}(z)=\frac{i}{2 \pi} \ln \left(1+\gamma \mathcal{V}^{(m)}(z)\right)
\end{array}
$$

There we have introduced a notation for the almost reduced functions

$$
\begin{gathered}
\mathcal{W}^{(m)}=\mathcal{W}_{1}\left(\begin{array}{c}
-m q \\
m q
\end{array}\right), \quad \mathcal{H}^{(m)}(z)=\mathcal{H}_{1}\left(\begin{array}{c}
-m q \\
m q
\end{array}\right), \quad \mathcal{V}^{(m)}(z)=\mathcal{V}_{1}\left(z \mid \begin{array}{c}
-m q \\
m q
\end{array}\right), \\
\text { as well as } \quad\left(\mathcal{G}^{(m)}\right)^{\prime}(z)=\partial_{\epsilon} \mathcal{G}_{2}\left(\begin{array}{c}
-m q, z \\
m q, z+\epsilon
\end{array}\right) .
\end{gathered}
$$

Proof - If one restricts oneself to the leading O(1) part of this action, then, as before, one should only consider the part of the sum corresponding to integers $k_{\ell}$ such that $\sum k_{\ell}=N-2$. All the others will produce subdominant contributions. Next, one should once again act with the derivatives on the exponent $\exp \left\{x \mathcal{G}_{n}\right\}$ exactly as it was done in (4.8).

Note that, for the oscillating functional we consider, there are at least 2 variables $z_{\ell}$. Moreover, after the computation of derivatives one should set $z_{1}=\cdots=z_{p}=\sigma q$ and $z_{p+1}=\cdots=z_{r}=-\sigma q, p \in[1 ; r-1]$. Indeed, for the slowest oscillating terms when a maximal number of derivatives $\Sigma k_{\ell}=N-2$ is considered, then there is only one jump in the sequence $\sigma_{\ell}$. 
The first step of the analysis, that is to say the determination of the action of the $\delta_{z \ell}^{(t)}\left(\mu_{\ell, t, j}\right)$ operators is the same as in the non-oscillating case. Then, in order to recover an $\mathrm{O}\left(x^{-2}\right)$ contribution, one should act with all of the derivatives only on the exponent $x \mathcal{G}_{n}$. The only difference in the proof consists in to the specifications of the $z$ 's. It is not difficult to see that, for any function satisfying the reduction properties (4.2):

$$
\mathcal{W}_{r}\left(\begin{array}{c}
\{\sigma q\}^{p} \cup\{-\sigma q\}^{r-p} \\
\{\sigma q\}^{p+\sigma m} \cup\{-\sigma q\}^{r-p-\sigma m}
\end{array}\right)=\mathcal{W}_{1}\left(\begin{array}{c}
-m q \\
m q
\end{array}\right) .
$$

Thence,

$$
\begin{aligned}
& x^{-N} I_{n}^{(N ; m)}\left[\mathcal{F}_{n}\right]= x^{-2} \mathcal{W}^{(m)} \mathrm{e}^{x \mathcal{G}^{(m)}+\ln x \mathcal{H}^{(m)}} \mathbb{S}_{N}^{(m)} * \sum_{\Sigma k_{\ell}=N-2} \\
& G_{\left\{k_{\ell}\right\}}^{\mathfrak{S}_{N}^{(m)}}\left\{\prod_{s=1}^{r}\left[\epsilon_{s} \mathcal{G}^{\prime}\left(z_{s}\right)\right]^{k_{s}}\left[\mathcal{V}^{(m)}\left(z_{s}\right)\right]^{p_{s 0}} \prod_{t=1}^{n}\left\{\int_{-q}^{q} \mathrm{~d} \mu \frac{\left[\mathcal{V}^{(m)}\left(z_{s}\right)\right]^{t}-\left[\mathcal{V}^{(m)}(\mu)\right]^{t}}{z_{s}-\mu}\right\}^{p_{\ell t}}\right\}+\mathrm{o}(1) .
\end{aligned}
$$

It is now clear that the above series is exactly of the type considered in the sum-rule (3.15).

\section{Conclusion}

In this article, we have given the explicit proof of the action of the functionals involved in the AE of cyclic integrals. The form of this action was used in [9] for the computation of the asymptotic behaviour of the generating function of the spin-spin correlation functions in integrable models. Our result was proven thanks to a precise determination of the structure of a part of the $\mathrm{AE}$ of $\ln \operatorname{det}[I+V]$ to any order in $x^{-N}$. These were obtained thanks to several equivalent ways of representing the kernel of the generalized sine kernel. We have only derived this action up to the first non-vanishing terms. However, the method that we have developed can also be applied, with the price of increasing complexity, to compute the sub-leading corrections to these terms. These, in turn would allow one to access to corrections in the long-distance asymptotic behavior of $\left\langle\mathrm{e}^{\beta Q_{x}}\right\rangle$ that deviate from the conformal field theory predictions.

\section{Acknowledgements}

Karol K. Kozlowski is supported by the ANR program GIMP ANR-05-BLAN-0029-01 and by the French ministry of research. The author would like to thank the Centre de recherche mathématiques of the Université de Montréal for its hospitality during the conference "Integrable quantum systems and solvable statistical models". The author is grateful to N.-Kitanine, J.-M. Maillet, N. A. Slavnov and V. Terras for many stimulating discussions.

\section{References}

[1] I. Affleck, Field theory methods and quantum critical phenomena, ed. E. Brézin and J. Zinn-Justin, NorthHolland, Amsterdam.

[2] H. E. Boos, M. Jimbo, T. Miwa, F. Smirnov, and Y. Takeyama, "Hidden Grassmann structure in the XXZ model.", Comm. Math. Phys. 272 (2007), 263-281. 
[3] J. L. Cardy, "Conformal invariance and universality in finite-size scaling.", J. Phys. A: Math. Gen. 17 (1984), L385-387.

[4] J.-S. Caux, P. Calabrese, and N.A. Slavnov, "One-particle dynamical correlations in the one-dimensional Bose gas .", J. Stat. Mech. (2007), P01008.

[5] J.-S. Caux, R. Hagemans, and J.-M. Maillet, "Computation of dynamical correlation functions of Heisenberg chains: the gapless anisotropic regime.", J. Stat. Mech. 95 (2005), P09003.

[6] F. D. M. Haldane, "General relation of correlation exponents and spectral properties of one-dimensional Fermi systems: Application to the anisotropic s = 1/2 Heisenberg chain.", Phys. Rev. Lett. 45 (1980), 13581362.

[7] M. Jimbo, T. Miwa, Y. Mori, and M. Sato, "Density matrix of an impenetrable Bose gas and the fifth Painlevé transcendent.", Physica D 1 (1980), 80-158.

[8] N. Kitanine, K. K. Kozlowski, J.-M. Maillet, N. A. Slavnov, and V. Terras, "On correlation functions of integrable models associated with the six-vertex R-matrix.", J. Stat. Mech. (2007), P01022.

[9] N. Kitanine, K. K. Kozlowski, J.-M. Maillet, N. A. Slavnov, and V. Terras, "Algebraic Bethe Ansatz approach to the asymptotics behavior of correlation functions.", math-ph/ 08080227 (2008).

[10] N. Kitanine, K. K. Kozlowski, J.-M. Maillet, N. A. Slavnov, and V. Terras, "The Riemann-Hilbert approach to a generalized sine kernel and applications.", math-ph/ 08054585 (2008).

[11] N. Kitanine, J.-M. Maillet, N.A. Slavnov, and V. Terras, "Master equation for spin-spin correlation functions of the XXZ chain.", Nucl.Phys. B 712 (2005), 600-622.

[12] N. Kitanine, J.-M. Maillet, and V. Terras, "Form factors of the XXZ Heisenberg spin-1/2 finite chain.", J. Phys. A: Math. Gen. 35 (2002), L753-10502.

[13] T. Kojima, V. E. Korepin, and N. A. Slavonv, "Determinant representation for dynamical correlation functions of the quantum nonlinear Schrödinger equation.", Comm. Math. Phys. 188 (1997), 657-689. 